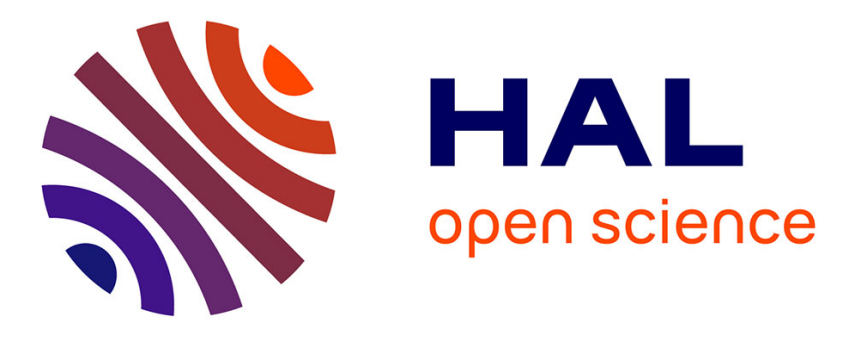

\title{
Shuffling of DNA cassettes in a synthetic integron
}

\author{
David Bikard, Didier Mazel
}

\section{To cite this version:}

David Bikard, Didier Mazel. Shuffling of DNA cassettes in a synthetic integron. Karen M. Polizzi; Cleo Kontoravdi. Synthetic Biology, 1073, Humana Press, pp.169-174, 2013, Methods in Molecular Biology (MIMB), 978-1-62703-624-5 (Hardcover), 978-1-4939-6286-0 (Softcover), 978-1-62703-625-2 (eBook). 10.1007/978-1-62703-625-2_14 . pasteur-01698800

\section{HAL Id: pasteur-01698800 https://hal-pasteur.archives-ouvertes.fr/pasteur-01698800}

Submitted on 1 Feb 2018

HAL is a multi-disciplinary open access archive for the deposit and dissemination of scientific research documents, whether they are published or not. The documents may come from teaching and research institutions in France or abroad, or from public or private research centers.
L'archive ouverte pluridisciplinaire HAL, est destinée au dépôt et à la diffusion de documents scientifiques de niveau recherche, publiés ou non, émanant des établissements d'enseignement et de recherche français ou étrangers, des laboratoires publics ou privés. 


\section{Shuffling of DNA cassettes in a synthetic integron}

\section{Summary}

The complexity of even small gene networks makes them difficulty amenable to rational design. Testing random combinations of genetic elements in a directed evolution procedure is thus of interest for many applications including metabolic engineering. Here we describe how the recombination machinery of class 1 integrons can be used to deliver and shuffle genetic elements at a chromosomal locus in E.coli.

Key words:

Synthetic Biology, Directed Evolution, Recombination, DNA shuffling, Integron, Metabolic Engineering.

\section{Introduction}

Over the past 20 years, efficient mutagenesis techniques have been developed allowing the introduction of random point mutations, as well as sequence shuffling through homologous recombination. However the efficient random recombination of heterologuous sequences largely remains a challenge. It has been achieved in vitro through various techniques of randomized assembly ligation and overlap extension PCR (1-5). However these techniques are limited in the size and number of elements they can combine.

Integron are natural recombination platforms involved in the rapid adaptation of bacteria to changing environment. They are present in the genome of roughly $10 \%$ of sequenced bacteria, and are largely responsible for the massive spread of multiple resistances to antibiotics. Integrons consists in an integrase gene (intI) able to capture, stockpile and shuffle gene cassettes. Natural cassettes generally consist in a single promoterless ORF associated to an attC recombination site. Cassettes can be excised through attC x attC recombination into a circular intermediate and preferentially integrated at a primary recombination site downstream of the integrase gene called attI. Different classes of integrons have been identified on the base of the integrase sequence identity (for a comprehensive review see (6)).

We use here the class 1 integrase isolated from R388 multiresistant integron, the associated $a t t I 1$ site, and the $a t t C_{a a d A 7}$ site isolated from an aadA7 spectinomycin resistance cassette. These elements have been extensively described and shown to efficiently recombine. We have already shown how this machinery can be used to deliver gene cassettes of the tryptophan operon and shuffle them to generate a wide variety of combination yielding improved phenotypes (7). Here is a detailed procedure of the steps required to construct a strain with a synthetic integron integrated in the chromosome, to deliver new genetic elements in the array and to shuffle them. 


\section{Materials}

The minimal platform of an integron consists in the integrase gene and the attI recombination site. In all experiments, the IntI 1 integrase is expressed in trans from the pBAD::intI1 plasmid. The attIl site is integrated into the chromosome at the attB site of bacteriophage lambda using a suicide integration vector (procedure described below). Depending on your application you may want to directly clone cassettes in the integron array that you will integrate into the chromosome.

\subsection{Biological material: DNA}

1. Plasmid pSWK carrying an attI site. The pSWK plasmid carries an R6K origin of replication which depends on the $\Pi$ protein expressed from the pir gene. It also carries the attP site from bacteriophage lambda, allowing its integration at the lambda $a t t B$ site in the E.coli chromosome. This plasmid complies with biobrick standard RFC10, and can be found on the registry of standard biological parts as BBa_J99000 (7).

2. The integron att $_{\text {aadA7 }}$ site: BBa_J99001 (7).

3. The integron attIl site: BBa_J99002 (7).

4. Plasmid pBAD::IntI1 (8).

5. Plasmid pTSA29-CXI-AK (9). A thermo-sensitive pSC101 vector carrying the lambda integrase under the control of the thermo-inducible CI857/pR promoter.

\subsection{Biological material: strains}

1. pil E.coli cells (DH5a AthyA::(erm-pir116) [EmR]), for the replication of the pSWK plasmid (10)

2. MG1655 E.coli cells (or any E.coli strain where you want to integrate the synthetic integron)

3. $\omega 7249$ E.coli cells ((F-) RP4-2-Tc::MuAnic35 AdapA::(erm-pir) [KmR ErmR]). $\omega 7249$ cells express the pir gene allowing the replication of vectors carrying the R6K replication origin. It also expresses the RP4 conjugation machinery allowing the transfer of plasmids carrying the RP4 origin of transfer (11).

\subsection{Culture media and reagents}

1. Luria Bertani (LB) culture medium

2. LB agar plates supplied with suitable selection agent

3. Antibiotics were used at the following concentrations: ampicillin (Ap), 100 $\mu \mathrm{g} / \mathrm{ml}$, chloramphenicol $(\mathrm{Cm}), 25 \mu \mathrm{g} / \mathrm{ml}$.

4. Thymidine (Thy) and diaminopimelic acid (DAP) is supplemented when necessary to a final concentration of $0.3 \mathrm{mM}$.

5. Glucose and L-arabinose are added at respectively 10 and $2 \mathrm{mg} / \mathrm{ml}$ final concentration for the repression and induction of the $\mathrm{pBAD}$ promoter.

6. $\mathrm{CaCl} 2$ solution, $50 \mathrm{uM}$

7. EcoRI, XbaI, SpeI, PstI restriction enzymes. Those are the restriction sites of the RFC10 assembly standard.

8. T4 DNA ligase 


\section{Methods}

The global idea is to first integrate the attIl site into the chromosome. This will be the landing platform of any integron cassette of your choice. Cassettes present in the array can be shuffled through the expression in trans of the IntI1 integrase from the pBAD::intI1. Finally, new cassettes can be delivered to the array trough a suicide conjugation assay.

Depending on your application an initial cassette array can directly be integrated together with the attI site. Cassettes are simply created through the association of any DNA element to an att $C_{a a d A 7}$ recombination site and cloned downstream of the attI site. This can be done using the construction method of your choice. The method we use here is biobrick standard assembly. We constructed vectors carrying attI (BBa_J99002) and attC sites (BBa_J99001) complying with the RFC10 standard.

Several methods have been described for the efficient integration of DNA in the E.coli chromosome $(12,13)$. The method we chose here is to clone the initial integron cassette array on a vector (pSWK) that can then be integrated at the chromosomal attB site of bacteriophage lambda. pSWK carries an attP site and can thus be integrated at the attB site by the lambda integrase. It has an R6K origin of replication and can only replicate in cells expressing the pir gene. This plasmid can thus be integrated into pircell expressing the lambda integrase in trans (from plasmid pTSA29-CXI-AK), and plating on the pSWK resistant marker will select integration events. However, any other integration method and any other neutral chromosomal locus should be suitable.

New cassettes can be delivered to an integron array through a suicide conjugation strategy. The pSWK vector can be used to this end too. pSWK carries an RP4 origin of transfer, and can be delivered to recipient cells by $\omega 7249$ cells expressing the RP 4 conjugation machinery. This donor strain can be used in a conjugation assay to deliver cassettes in a recipient strain carrying an integron array and expressing the IntI1 integrase in trans from the pBAD::IntI1.

\subsection{Construction of the initial cassette array}

1. The initial integron array can be constructed with the method of your choice. Here, we use a classic digestion / ligation / transformation protocol. The use of the RFC10 idempotent assembly standard allows the easy association of any biobrick to an att $_{a a d A 7}$ site and its cloning into the integron array on the pSWK vector. For more details on biobrick assembly see partsregistry.org. The pSWK vector can only replicate in cells expressing the pir gene. Transformation is thus carried out into pil cells, and selection on LB agar + Chloramphenicol $(25 \mathrm{microg} / \mathrm{ml})+$ Thymidine $(70 \mathrm{microg} / \mathrm{ml})$ plates.

\subsection{Integration of the initial cassette array into the chromosome}

1. You first need to transform the pTSA29-CXI-AK plasmid carrying the lambda integrase into your strain. The method of your choice can be used to prepare competent cells. We use here a classical $\mathrm{CaCl} 2$ chemo-competent protocol. After heat-shock, cells need to be incubated at $30^{\circ} \mathrm{C}$ for the expression period 
and plated on $\mathrm{LB}$ agar $+\mathrm{Ap}$ at $30^{\circ} \mathrm{C}$ in order for the plasmid to stably replicate.

2. In order to integrate your pSWK into these cells, you now need to prepare competent cells expressing the lambda integrase. Launch an overnight culture at $30^{\circ} \mathrm{C}$ in $\mathrm{LB}+\mathrm{Ap}$ from a single colony

3. Dilute the overnight culture to the $1 / 100^{\text {th }}$ in $\mathrm{LB}$ and grow at $30^{\circ} \mathrm{C}$ until $\mathrm{OD}_{600}=0.2$

4. Switch the incubation temperature to $42^{\circ} \mathrm{C}$ until $0.6<\mathrm{OD}_{600}<0.8$. During this period, the lambda integrase will be expressed from the CI857-PR promoter.

5. Prepare $\mathrm{CaCl} 2$ competent cells (or any other type of competent cells. The integration event is very efficient, so you don't need to prepare ultracompetent cells for this to work). (see note 1 )

6. Transform with at least $20 \mathrm{ng}$ of pSWK DNA, and incubate for the expression time for $1 \mathrm{H}$ at $42^{\circ} \mathrm{C}$.

7. Plate on $\mathrm{LB}$ agar $+\mathrm{Cm}$ and grow overnight at $42^{\circ} \mathrm{C}$. Doing all this steps at $42^{\circ} \mathrm{C}$ ensures that the lambda integrase is expressed and that at the same time the pTSA29 is lost.

8. The following day you should have colonies with the pSWK integrated at the attB site of your strain.

9. Check for proper integration by doing a colony PCR using a primer binding on the pSWK and one binding on the chromosome.

\subsection{Cassette shuffling through the IntI1 integrase expression}

1. Transform the pBAD::IntI1 in your cells using the method of your choice.

2. Induce the integrase by diluting an overnight culture to the $1 / 100^{\text {th }}$ in $\mathrm{LB}+$ Ara. You can prepare a negative control in LB + Glu. Grow overnight (see note 2)

3. Plate cells on the selective media of your choice. Recombinations induced by the integron machinery happen at frequencies below $10^{-2}$. In order to find recombined clones, you need a powerful screening assay. In our initial study where we shuffle the genes of the tryptophan biosynthesis operon, the selection is performed in tryptophan auxotroph strains and recombinants are selected on minimal media (7).

\subsection{Delivery of new cassettes through conjugation}

1. Transform the pSWK carrying the cassettes of interest into $\omega 7249$ cells. We use $\mathrm{CaCl} 2$ competent cells, but any method is suitable. Remember that $\omega 7249$ cells need DAP to grow. (see note 3 )

2. This strain will be your donor strain in the conjugation assay. Depending on your application you may want to use several donor strains to combinatorially deliver cassettes of interest in a single recipient strain. The recipient strain needs to carry the pBAD::intI1 plasmid and an attI site in the chromosome. Dilute an overnight culture of the donor strain(s) to the $1 / 100^{\text {th }}$ in $\mathrm{LB}+\mathrm{DAP}$ and of the recipient strain in $\mathrm{LB}+\mathrm{DAP}+\mathrm{Ara}$ (see note 4).

3. Grow donor and recipient cells to $0.4<\mathrm{OD}_{600}<0.6$

4. Mix $1 \mathrm{ml}$ of donor cells with $4 \mathrm{ml}$ of recipient cells and filter onto a $0.22 \mathrm{um}$ membrane. (see note 5) 
5. Place the filter on a fresh $\mathrm{LB}+\mathrm{DAP}+$ Ara plate, the side with the cells facing up. Incubate $6 \mathrm{H}$ at $37^{\circ} \mathrm{C}$.

6. Put the filter in a $50 \mathrm{ml}$ tube with $5 \mathrm{ml} \mathrm{LB}$ and vortex to remove the cells from the filter.

7. Plate the cells on the selective media of your choice. (If the pSWK vector you use carries a resistance that is not already present in your recipient strain, you can select for the integration of the whole vector in the integron array. It is then possible to get rid of the vector backbone using a counter selection marker, leaving all or some of the cassettes in the chromosome.)

\section{Notes}

1. It is important that competent cells are kept on ice throughout the whole process.

2. The longer the cells are growing with the integrase expressed, the higher the recombination frequency will be. To improve recombination frequency, several passages in LB+Ara can be done. Note however that without selection, cassettes are lost at a frequency similar to the one of reordering events (7).

3 . The use of the DAP auxotroph donor $(\omega 7249)$ for the conjugation delivery protocol guaranties that you get rid of the donor by simply selecting on LB + antibiotic marker of the pSWK, as DAP is completely absent in LB.

4. The recipient strain does not actually need DAP to grow, but adding DAP in the medium allows to later mix the cultures without worrying about killing the donor cells

5. If you do not have the equipment to do this, you can mix the cells and centrifuge them gently $4 \mathrm{~min}$ at $2000 \mathrm{~g}$, discard the supernatant, resuspend the cells in 100 microl and pipette onto the filter.)

1. Bittker JA, Le BV, Liu JM, \& Liu DR (2004) Directed evolution of protein enzymes using nonhomologous random recombination. Proc Natl Acad Sci U $S$ A 101(18):7011-7016.

2. Gertz J, Siggia ED, \& Cohen Ba (2009) Analysis of combinatorial cisregulation in synthetic and genomic promoters. Nature 457(7226):215.

3. Guet CC, Elowitz MB, Hsing W, \& Leibler S (2002) Combinatorial synthesis of genetic networks. Science 296(5572):1466.

4. Cox RS, Surette MG, \& Elowitz MB (2007) Programming gene expression with combinatorial promoters. Molecular systems biology 3:145.

5. Tsuji T, Onimaru M, \& Yanagawa H (2001) Random multi-recombinant PCR for the construction of combinatorial protein libraries. Nucleic Acids Res 29(20):E97.

6. Cambray G, Guerout AM, \& Mazel D (2010) Integrons. (Translated from eng) Annu Rev Genet 44:141-166 (in eng). 
7. Bikard D, Julie-Galau S, Cambray G, \& Mazel D (2010) The synthetic integron: an in vivo genetic shuffling device. (Translated from Eng) Nucleic Acids Res (in Eng).

8. Demarre G, Frumerie C, Gopaul DN, \& Mazel D (2007) Identification of key structural determinants of the IntI1 integron integrase that influence attC $\mathrm{x}$ attI1 recombination efficiency. Nucleic Acids Res 35(19):6475-6489.

9. Valens M, Penaud S, Rossignol M, Cornet F, \& F. B (2004) Macrodomain organization of the Escherichia coli chromosome. EMBO Journal 23(21):4330-4341.

10. Demarre G, et al. (2005) A new family of mobilizable suicide plasmids based on the broad host range R388 plasmid (IncW) or RP4 plasmid (IncP $\alpha$ ) conjugative machineries and their cognate E.coli host strains. Research in microbiology 156(2):245-255.

11. Babic A, GuÃCrout A-M, \& Mazel D (2008) Construction of an improved RP4 (RK2)-based conjugative system. Research in microbiology 159(78):545.

12. Datsenko KA \& Wanner BL (2000) One-step inactivation of chromosomal genes in Escherichia coli K-12 using PCR products. (Translated from eng) Proc Natl Acad Sci U S A 97(12):6640-6645 (in eng).

13. Chaveroche MK, Ghigo JM, \& d'Enfert C (2000) A rapid method for efficient gene replacement in the filamentous fungus Aspergillus nidulans. Nucleic Acids Res 28(22):E97. 